\title{
Disseminated Blastomycosis Presenting with Spontaneous Coronary Artery Dissection
}

\author{
Rahul Sehgal $^{1}$, D Fearghas O'Cochlain ${ }^{2}$, Andrew R Virata ${ }^{3}$, Gurpreet Singh ${ }^{2}$ \\ ${ }^{1}$ Department of Rheumatology, Mayo Clinic Alix School of Medicine, Mayo Clinic Health System, Eau Claire, Wisconsin, USA \\ 2 Department of Cardiovascular Medicine, Mayo Clinic Alix School of Medicine, Mayo Clinic Health System, Eau Claire, Wisconsin, USA \\ ${ }^{3}$ Department of Pathology, Mayo Clinic Alix School of Medicine, Mayo Clinic Health System, Eau Claire, Wisconsin, USA
}

\section{Doi: 10.12890/2021_002511- European Journal of Case Reports in Internal Medicine - ( ) EFIM 2021}

Received: 24/03/2021
Accepted: 30/03/2021
Published: 23/04/2021

How to cite this article: Sehgal R, O'Cochlain DF, Virata AR, Singh G. Disseminated blastomycosis presenting with spontaneous coronary artery dissection. EJCRIM 2021;8: doi:10.12890/2021_002511.

Conflicts of Interests: All authors declare no competing interests.

This article is licensed under a Commons Attribution Non-Commercial 4.0 License

\section{ABSTRACT}

Spontaneous coronary artery dissection (SCAD) is increasingly recognized as an important cause of acute coronary syndrome (ACS) and myocardial infarction (MI) in individuals with few or no known atherosclerotic risk factors. While systemic autoimmune inflammatory disorders are associated with precipitating SCAD, the role of infection-induced systemic inflammation in SCAD is not well defined. We present the case of a 49-year-old Caucasian woman with ST-elevation myocardial infarction (STEMI) diagnosed as SCAD from a severe systemic inflammatory response related to disseminated blastomycosis. Punch biopsy of a skin lesion and synovial fluid culture confirmed Blastomyces dermatitidis. This case suggests the possibility of systemic infection-induced inflammation as a precipitating factor in SCAD pathogenesis similar to autoimmune inflammatory disorders.

\section{LEARNING POINTS}

- Recognize the role of systemic inflammation from severe infection as a possible cause of spontaneous coronary artery dissection (SCAD).

- Recognize that cardiac involvement is rare in blastomycosis.

- Coronary revascularization may be required in SCAD for haemodynamic instability, ischaemic chest pain progression, and myocardium at risk.

\section{KEYWORDS}

Spontaneous coronary artery dissection, ST-elevation myocardial infarction, blastomycosis

\section{CASE DESCRIPTION}

A 49-year-old Caucasian woman with a medical history of hypertension presented to the emergency department with acute onset of crushing substernal chest pain, cough and dyspnoea. In addition to chest pain, the patient had experienced 4 weeks of progressively worsening malaise, left ankle pain, and swelling. In addition, she developed an asymptomatic, well-demarcated nodular lesion with an erythematous base on the right index finger (Fig. 1). She had received treatment with doxycycline 2 weeks prior to presentation for a presumed diagnosis of Lyme infection without improvement.

An admission electrocardiogram (ECG) showed inferior ST-segment elevation with reciprocal changes. Troponin T was elevated at 17 ( $\leq 10$ $\mathrm{ng} / \mathrm{l})$. The patient had considerable chest discomfort and haemodynamic instability that prompted an emergent percutaneous coronary 


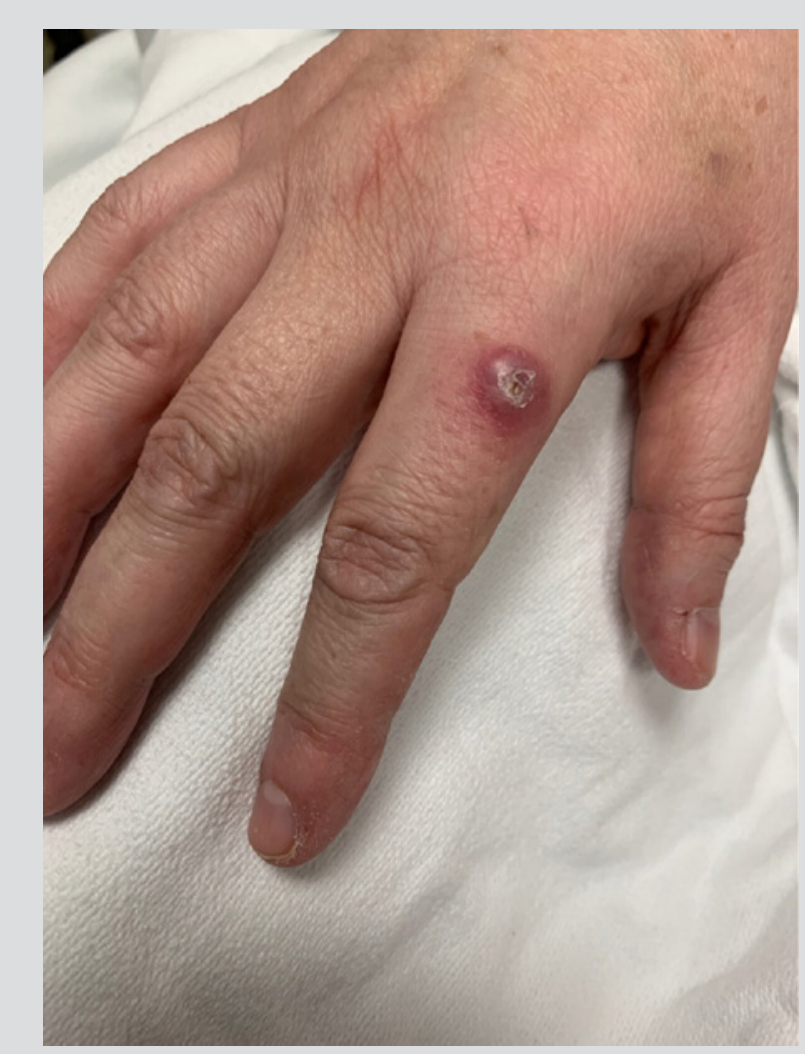

Figure 1. Skin lesion: deeply erythematous, well-demarcated lesion with a superficial healing erosion on the dorsum of the right index finger

intervention ( $\mathrm{PCl}$ ). The proximal and mid-right coronary artery (RCA) was diffusely tapered with an 80\% proximal lesion (Fig. 2). There was suspicion of an intramural haematoma as the cause of vessel narrowing. The proximal and mid-RCA was stented which caused propagation of the intramural haematoma to the distal RCA (Fig. 3). Intravascular ultrasonography (IVUS) showed an intramural haematoma with compression of the lumen more distally and a third stent was advanced over the overlap in the mid-RCA with good results (Fig. 4). Later, an echocardiogram showed a normal left ventricular ejection fraction (LVEF) without regional wall motion abnormalities, valvular heart disease or pericardial effusion.

A complete blood count was significant for leucocytosis of $12.6\left(3.4-9.6 \times 10^{9} / \mathrm{l}\right)$, erythrocyte sedimentation rate (ESR) of 88 (0-22 $\mathrm{mm} / \mathrm{hr}$ ) and C-reactive protein (CRP) of $110(<8 \mathrm{mg} / \mathrm{l})$.

The next day, the patient continued to remain lethargic with worsening pain in the left ankle joint. Arthrocentesis from the left ankle yielded $5 \mathrm{ml}$ of cloudy fluid with 15,000 white blood cells and $87 \%$ neutrophils without evidence of crystals. Bacterial and fungal cultures were performed on the synovial fluid.

A punch biopsy of the right index finger skin lesion was performed. Computed tomography of the chest, abdomen and pelvis revealed diffuse, miliary pulmonary nodules in both lungs. Magnetic resonance imaging of the brain showed scattered, punctate enhancing lesions throughout the brain.

Enzyme immunoassay for Blastomyces, Aspergillus, Coccidioides, Cryptococcus and Histoplasma from blood and urine were negative. A QuantiFERON Gold test for latent tuberculosis and nasopharyngeal swab for COVID-19 antigen and a COVID-19 antibody test were negative.

The punch biopsy from the finger lesion demonstrated an inflammatory infiltrate composed of neutrophils, eosinophils, lymphocytes, histiocytes, and broad-based budding yeast forms consistent with Blastomyces dermatitidis (Fig. 5). Synovial fluid culture from the left ankle was positive for a filamentous fungus identified as B. dermatitidis/gilchristii after 10 days of incubation.

The patient started treatment with intravenous liposomal amphotericin B $5 \mathrm{mg} / \mathrm{kg}$ daily for 6 weeks followed by oral itraconazole $200 \mathrm{mg}$ daily for 6 months to treat disseminated blastomycosis. Incision and drainage of the left ankle joint was performed.

The patient continued to improve on antifungal treatment, and at 1-month follow-up, her joint pains, malaise and skin lesions had resolved without recurrence of chest pain. The ESR and CRP normalized, and repeat imaging of the chest showed resolution of miliary pulmonary nodules. 


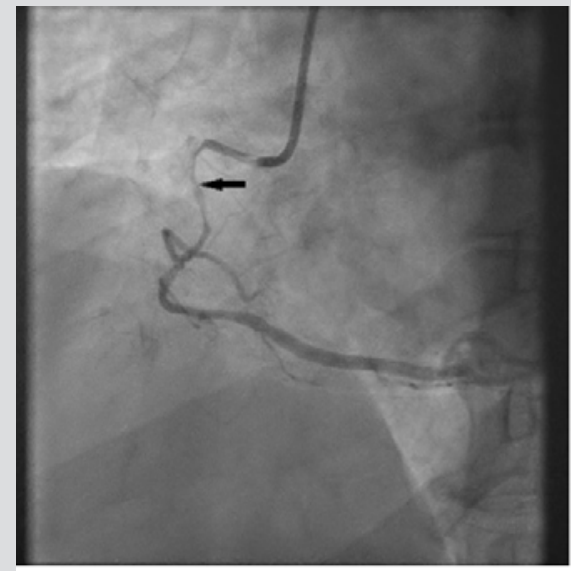

Figure 2. Spontaneous coronary artery dissection (SCAD) of the proximal right coronary artery (RCA): coronary angiography shows SCAD causing near occlusion of the proximal and mid portions of the RCA (arrow)

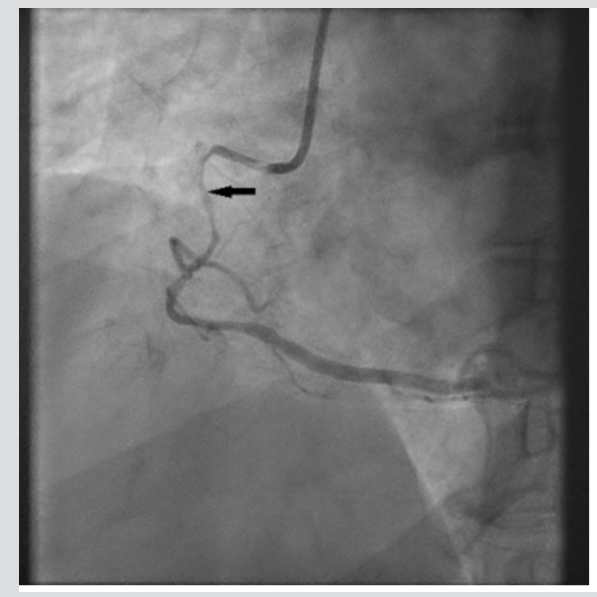

Figure 3. Extension of the right coronary artery (RCA) intramural haematoma: coronary angiography after stenting of the proximal and mid RCA shows the extending intramural haematoma (arrow)

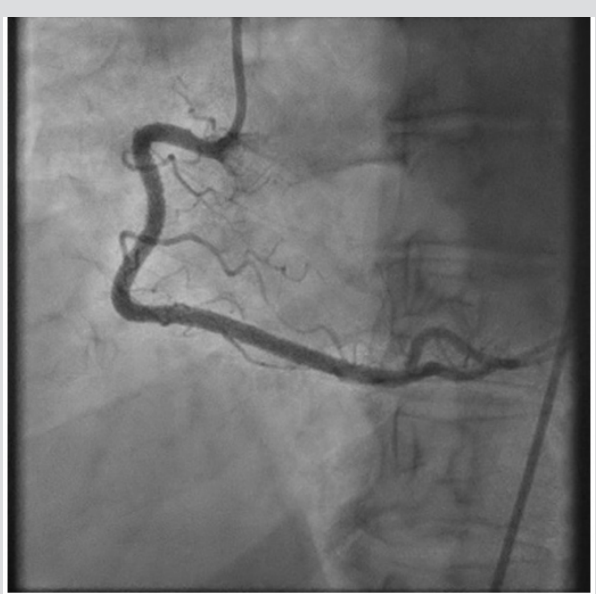

Figure 4. Post right coronary artery (RCA) stent with normal flow: coronary angiography after deployment of multiple stents in the RCA shows restoration of normal blood flow

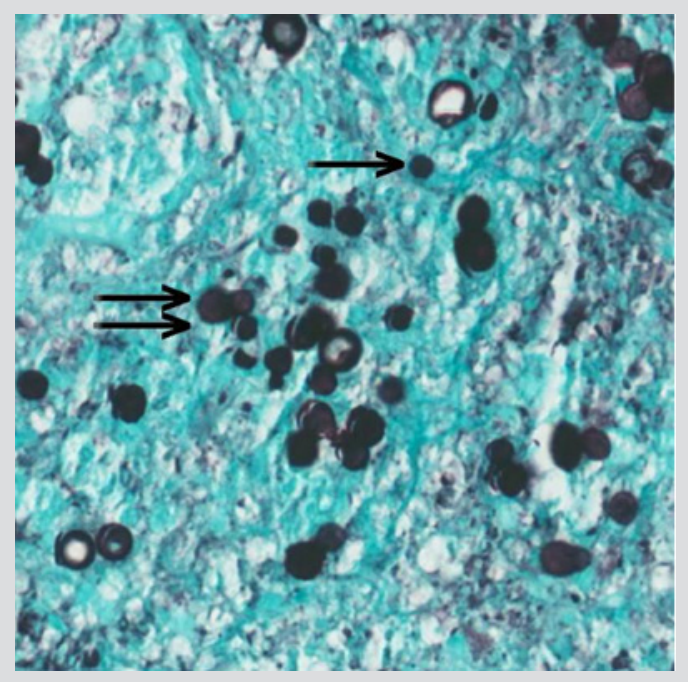

Figure 5. Skin biopsy from the finger lesion: Grocott methenamine silver (GMS) stain, intermediate power (20x magnification), stains numerous single (single arrow) and broadbased budding (double arrow) yeast forms

\section{DISCUSSION}

The cause of spontaneous coronary artery dissection (SCAD) (or epicardial coronary artery dissection) is unknown, although several inflammatory disorders, fibromuscular dysplasia, stimulant medications, illicit drugs and hormonal triggers (pregnancy) have been associated with its onset ${ }^{[1]}$. Case reports describe an association between SCAD and inflammatory disorders such as systemic lupus erythematosus, sarcoidosis, inflammatory bowel disease and coeliac disease, indicating the role of systemic inflammation in SCAD pathogenesis ${ }^{[2]}$. In recent times, increasing recognition of SCAD occurrence in COVID-19 patients suggests the role of systemic inflammation in response to infection [3]. Proposed mechanisms for SCAD in acute systemic infections include increased activation and infiltration of T-cells in the adventitia and peri-adventitial fat, which leads to overproduction of cytokines and proteases, increasing the risk of plaque rupture and dissection (insideout mechanism of SCAD) ${ }^{[4]}$. On the other hand, severe systemic infections may also stimulate angiogenesis and vasa vasorum proliferation, which has a greater tendency to disrupt (weak vessels) and develop intramural haematoma (outside-in mechanism of SCAD) ${ }^{[5]}$. Pathological biopsy specimens of dissected epicardial coronary arteries in SCAD show inflammation limited to adventitia and peri-adventitial soft tissue instead of transmural inflammation (the latter is seen in systemic vasculitis). Emotional and physical stress from severe infection can also cause a catecholamine surge that can act as an inciting trigger. SCAD occurs in $0.5 \%$ of acute coronary syndrome (ACS) patients and manifests as myocardial infarction (MI) in most of the affected individuals, with smaller percentages manifesting as ventricular arrhythmia and cardiogenic shock. 
Blastomycosis is a fungal infection endemic to regions of the Great Lakes, Mississippi and Ohio River valleys and is hyperendemic in northcentral Wisconsin. Blastomycosis commonly affects immune-competent persons, although in immunocompromised persons the disease tends to be more severe. In more than $50 \%$ of blastomycosis cases, the pulmonary disease may remain subclinical, and disease severity can vary from a mild illness to disseminated disease (25-50\% of cases). Involvement of the heart in disseminated blastomycosis is rare and difficult to diagnose. Blastomycosis-associated endocarditis can be especially challenging to diagnose in immunocompetent individuals as most biopsy-proven endocarditis cases have negative blood cultures. Cardiac blastomycosis involving the pericardium, myocardium and endocardium has only been reported on autopsy studies due to difficulty establishing this diagnosis ante mortem. With early recognition and advances in antifungal therapy, severe cardiac blastomycosis and endocarditis are exceedingly rare. Nonetheless, disseminated blastomycosis in the acute phase is a severe inflammatory disease with heightened emotional and physical stress and systemic inflammatory response that can precipitate SCAD. Stress catecholamine surge and inflammatory cell infiltration during systemic infection has the potential to increase coronary artery shear stress, and coronary dissection (inside-out mechanism).

Identification of broad-based budding yeast and DNA probe assays can lead to early diagnosis when a severely ill patient is encountered. The systemic inflammatory response accompanying disseminated blastomycosis can also result in the development of acute respiratory distress syndrome (ARDS), the most severe and potentially fatal complication of blastomycosis.

\section{CONCLUSION}

To our knowledge, this is the first case report of SCAD in an individual with disseminated blastomycosis. Our case does not distinguish causality from co-occurrence of disseminated blastomycosis and SCAD. However, the known association of systemic autoimmune inflammatory disorders and SCAD highlights the role of inflammation and heightened stress from systemic infection as a precipitating factor in SCAD. Our case further emphasizes the significance of evaluating inflammatory disorders (systemic infections or autoimmune diseases) in work-up of SCAD.

\section{REFERENCES}

1. Kim ESH. Spontaneous coronary-artery dissection. N Engl J Med 2020;383(24):2358-2370.

2. Saw J, Aymong E, Sedlak T, Buller CE, Starovoytov A, Ricci D, et al. Spontaneous coronary artery dissection: association with predisposing arteriopathies and precipitating stressors and cardiovascular outcomes. Circ Cardiovasc Interv 2014;7(5):645-655.

3. Shojaei F, Habibi Z, Goudarzi S, Firouzabadi FD, Montazerin SM, Najafi H, et al. COVID-19: a double threat to takotsubo cardiomyopathy and spontaneous coronary artery dissection? Med Hypotheses 2021;146:110410.

4. Adlam D, Alfonso F, Maas A, Vrints C. European Society of Cardiology, Acute Cardiovascular Care Association, SCAD Study Group: a position paper on spontaneous coronary artery dissection. Eur Heart J 2018;39:3353-3368.

5. Pitliya A, Datta S, Kalayci A, Kahe F, Sharfaei S, Jafarizade M, et al. Eosinophilic inflammation in spontaneous coronary artery dissection: a potential therapeutic target? Med Hypotheses 2018;121:91-94. 